\title{
Prevalencia de la infección genital por Chlamydia trachomatis en mujeres que asisten al Instituto Nacional de Perinatología de la Ciudad de México
}

\author{
Marcela López-Hurtado', Selene García-Romero', Marcos R. Escobedo-Guerra', \\ David Bustos-López' y Fernando M. Guerra-Infante',2
}

\section{Prevalence of genital Chlamydia trachomatis infection in women attending in the National Institute of Perinatology from Mexico City}

Background: Endocervical infection by Chlamydia trachomatis is considered one of the leading causes of infertility worldwide. During pregnancy, it can lead to serious complications such as premature rupture of membranes and premature births. Aim: To determine the prevalence of genital infection by $C$. trachomatis in pregnancy and infertile women from Mexico City. Methods: The detection of C. trachomatis was performed by real-time PCR with the commercial kit COBAS ${ }^{\circledR}$ TaqMan CT Test v2.0 (Roche Molecular System). Results: We analyzed 2,352 endocervical swabs; 102 were positive (4.3\%). Age prevalence showed that pregnant adolescents (15 to 19 years of age) had the highest risk of infection $(10.9 \%, \mathrm{RR}=3.23$ [95\% IC: $1.79-5.84])$, followed by young women aged 20 to 24 years, with a prevalence of $5.6 \%(\mathrm{RR}=1.65$ [95\% IC: $0.82-3.34])$. Discussion: The results indicate that the prevalence is within the range reported worldwide. However, pregnant adolescents were those with a higher prevalence than infertile women were. Conclusion: A systematic screening of C. trachomatis infection in women younger than 24 years of age, and in pregnant women is necessary to reduce the incidence of infertility and perinatal complications.

Key words: Chlamydia trachomatis; prevalence; pregnant women; teenagers; Mexico.

Palabras clave: Chlamydia trachomatis; prevalencia; embarazo; adolescentes; México.

\section{Introducción}

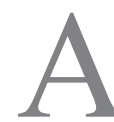

ctualmente, Chlamydia trachomatis es considerada como uno de los microorganismos más frecuentes que causan infecciones de transmisión sexual (ITS) ${ }^{1}$. En México, su prevalencia no se conoce por completo, debido a que no todos los estados de la República Mexicana llevan a cabo este estudio. En Ciudad de México se informa una prevalencia de infección genital de 1,5 al $28,9 \%$ en diferentes poblaciones femeninas ${ }^{2-4}$, mientras que, por serología, la prevalencia es mucho mayor, llegando hasta $38,3 \%$ en mujeres infértiles con oclusión tubárica, $35,4 \%$ en pacientes infértiles sin oclusión tubárica y $22,6 \%$ en mujeres embarazadas 5 . En algunos estados como Jalisco, Morelos, Oaxaca, Sonora, Tabasco y Yucatán, donde se ha realizado esporádicamente este diagnóstico, se informa de una prevalencia genital entre 1,5 y $14,5 \%{ }^{6-11}$. En cuanto a las trabajadoras sexuales se ha informado una prevalencia de 14,4 a 16,6\% en los Estados de Chiapas y Durango ${ }^{12,13}$. A pesar de lo anterior, la prevalencia de infección genital por $C$. trachomatis en mujeres mexicanas embarazadas (adultas y adolescentes) es aún desconocida.

Cabe señalar que, si la infección por C. trachomatis no es tratada con prontitud, las secuelas producidas por este patógeno son la enfermedad inflamatoria pélvica (EIP), infertilidad por oclusión tubárica y dolor pélvico crónico ${ }^{1}$. En mujeres embarazadas puede provocar embarazos ectópicos, nacimientos prematuros y nacimiento de bebés con neumonía, conjuntivitis o sepsis ${ }^{1,14}$.

Desde 1990, el Instituto Nacional de Perinatología (INPer) ha realizado un diagnóstico rutinario de la infección por $C$. trachomatis a diferentes pacientes que son aceptadas en el INPer por causas de infertilidad, pérdida gestacional recurrente y embarazos de alto riesgo, tanto para la salud de la madre como del bebé (adolescentes, con infección o con enfermedades autoinmunes, metabólicas y cardiovasculares, así como pacientes que entran al programa de climaterio).

Los métodos que se han empleado para este diagnóstico han sido: inmunoensayo enzimático, inmunofluorescencia, cultivo celular, hibridación en fase líquida y amplificación de ácidos nucleicos ${ }^{2,4}$. Todos estos métodos tienen una sensibilidad de 60 a $80 \%$ y una especificidad de 70 a $80 \%$, excepto la prueba de amplificación de ácidos nucleicos, cuya sensibilidad y especificidad son cercanas a $98 \% \%^{1,15}$.
'Instituto Nacional de Perinatología. Departamento de Infectología Laboratorio de Biología Molecular. ${ }^{2}$ Escuela Nacional de Ciencias Biológicas del IPN.

Departamento de Microbiología.

Los autores aquí firmantes declaran que no existe conflicto de intereses.

Fuente de financiamiento: No existe.

Recibido: 23 de diciembre de 2016

Aceptado: 22 de mayo de 2018

Correspondencia a: Fernando M. Guerra-Infante fguerra_96@yahoo.com 
El objetivo de este estudio es informar sobre la prevalencia de la infección por $C$. trachomatis en mujeres embarazadas e infértiles que acudieron al INPer en la Ciudad de México.

\section{Materiales y Métodos}

\section{Pacientes}

De enero a diciembre de 2015 se realizó un estudio prospectivo en mujeres que asistieron al INPer en la ciudad de México por causas de infertilidad o embarazo de alto riesgo. Las pacientes embarazadas seleccionadas fueron aquellas que mostraran flujo vaginal anormal, mientras que las mujeres infértiles elegibles fueron sintomáticas y asintomáticas de infección endocervical.

\section{Criterios de exclusión}

- Para ambos grupos, que fueran positivas para el aislamiento y la identificación de bacterias y levaduras como Neisseria gonorrhoeae, Mycoplasma hominis, Ureaplasma urealyticum, Gardnerella vaginalis, Streptococcus agalactiae, Trichomonas vaginalis y Candida albicans.

- La realización de ducha vaginal que antecediera a la toma de la muestra cervical.

- Que hubieran recibido terapia antimicrobiana sistémica o local previa a la evaluación.

- Que hubieran tenido relaciones sexuales $48 \mathrm{~h}$ antes de la toma de la muestra.

\section{Definiciones}

Infertilidad: Incapacidad temporal o definitiva de la pareja sexual para concebir un producto viable después de un año de relaciones sexuales sin el uso de un método de planificación familiar.

Flujo vaginal anormal: Se definió según el tiempo de inicio y duración del flujo, color, olor y consistencia, así como factores asociados: prurito, dispareunia, dolor abdominal, sangrado vaginal anormal y fiebre.

\section{Muestreo endocervical}

La muestra endocervical se obtuvo mediante la introducción de un hisopo de alginato de calcio en la cavidad vaginal para obtener un raspado epitelial cervical. Posteriormente, los hisopos se colocaron en un medio de transporte (System Copan Universal Transport Medium, UTM-RT). Este medio de transporte es útil para especímenes clínicos que contengan virus, Chlamydia, Mycoplasma y Ureaplasma (Copan Diagnostic Inc. Corona, CA, EUA). Las muestras contenidas en el medio de transporte fueron enviadas al laboratorio de virología donde se realizó el estudio de detección de C. trachomatis.

\section{Detección de C. trachomatis}

Se realizó utilizando el ensayo de amplificación de ácidos nucleicos en tiempo real, para ello se empleó el kit comercial COBAS ${ }^{\circledR}$ TaqMan CT Test v2.0 (Roche Molecular System, Inc., NJ, EE.UU.). La amplificación y detección automática se llevó a cabo en el analizador $\mathrm{COBAS}^{\circledR}$ TaqMan $^{\circledR} 48$. Este ensayo consiste en la amplificación de una proteína de la membrana externa principal del fragmento génico (182 pb) y del plásmido críptico (206 pb). La identificación de estas partículas se realizó mediante sondas marcadas con colorantes fluorescentes que se detectaron mediante reacción de polimerasa en cadena en tiempo real (RPC-TR).

\section{Recopilación de datos}

La edad y las causas por las cuales en la paciente se realizó el estudio de $C$. trachomatis se obtuvieron mediante el interrogatorio directo de éstas. Posteriormente, estos datos se confirmaron al revisar su expediente clínico.

\section{Análisis estadístico}

Para el análisis de datos se utilizaron medidas de tendencia central, dispersión y magnitud de la asociación. Para ello, se utilizó el programa estadístico SPSS para Windows (IBM Corporation, New Orchard Road, Armonk, NY, EUA, Versión 17.0.1, 2008).

Esta investigación fue aprobada por el Comité de Ética del INPer, de acuerdo con las normas éticas establecidas en la Declaración de Helsinki de 1964 y enmiendas posteriores.

\section{Resultados}

Durante el 2015 se realizaron 37.169 consultas por causas ginecológicas y obstétricas en el INPer. De éstas, 6.827 fueron mujeres infértiles, 957 mujeres en edad materna de riesgo y 610 mujeres embarazadas con infecciones de transmisión sexual (ITS).

El número de muestras endocervicales analizadas fue de 7.578. En 5.215 se identificó algún microorganismo y fueron tratadas con sus respectivos antimicrobianos (datos no mostrados) por lo que fueron excluidas del estudio.

Las 2.363 restantes fueron negativas al aislamiento microbiológico y fueron analizadas para el diagnóstico de infección por $C$. trachomatis. De éstas, 11 muestras fueron descartadas debido a que correspondían a mujeres que pertenecían a la clínica de climaterio.

La positividad para C. trachomatis fue de 102/2.352, con una prevalencia de 4,3\% (Tabla 1 ).

La edad media de las mujeres estudiadas fue de 29,6 $\pm 6,9$. La edad mínima fue de 12 años y la edad máxima, de 43 años. Cabe señalar que todas las adolescentes (12-19 años) eran mujeres embarazadas con infección cervicovaginal. 


\begin{tabular}{|c|c|c|c|c|c|c|}
\hline \multicolumn{7}{|c|}{ Chlamydia trachomatis } \\
\hline & & $\mathbf{n}$ & Positivo & Negativo & IC $95 \%$ & $p<$ \\
\hline \multirow[t]{2}{*}{ Mujeres embarazadas } & Adolescentes & 271 & $28(4,71 \%)^{b}$ & 243 & $2,9(1,53-5,48)$ & 0,003 \\
\hline & Adultas & 323 & $12(2,02 \%)$ & 311 & $1,0(0,52-1,74)$ & $N S^{a}$ \\
\hline Mujeres infértiles & Adultas & 1.758 & $62(3,53 \%)$ & 1.696 & Grupo control & \\
\hline
\end{tabular}

De las muestras analizadas, 1.758 fueron de mujeres infértiles y 594 fueron mujeres embarazadas (incluidas las adolescentes). La prevalencia de infección por $C$. trachomatis en ambos grupos fue de 3,53 y 6,73\%, respectivamente (Tabla 1). Las adolescentes embarazadas fueron las de mayor riesgo de adquirir la infección por C. trachomatis ( $\mathrm{RR}=2,9$ [ IC del 95\% 1,53-5,48]) en comparación con las adultas embarazadas.

La prevalencia de infección por edad se muestra en la Tabla 2. El grupo de mujeres de 15 a 19 años de edad presentó una prevalencia de $10,9 \%$ y fue estadísticamente significativo ( $\mathrm{RR}=3,23$; [IC del 95\% 1,79-5,84]; $\mathrm{p} \leq 0,001)$ en comparación con otros grupos de edad. Las adultas jóvenes de 20 a 24 mostraron una prevalencia de $5,6 \%(\mathrm{RR}=1,65$ [IC del 95\%: 0,82-3,34]).

El grupo con mayor número de participantes fue mujeres entre 30 y 34 años de edad; la prevalencia de infección por $C$. trachomatis en este grupo fue de $3,2 \%$, mientras que en mujeres de entre 35 y 39 años la prevalencia fue de $3,4 \%$ y en mujeres con edades de 25 a 29 años fue de 3,6\%, El grupo con menor prevalencia fue el de las mujeres sobre 39 años $(2,5 \%)$.

\section{Discusión}

En todo el mundo se ha informado de un aumento en la prevalencia de infección por C. trachomatis. La OMS ha documentado que la prevalencia mundial de $C$. trachomatis en el año 2012 fue de $4,2 \%{ }^{16}$. Los resultados de prevalencia obtenidos en este estudio muestran que los porcentajes están dentro del rango reportado por la OMS; sin embargo, esta prevalencia puede variar según las diferentes regiones entre 1,8 y $7,6^{1,16,17}$, así como por el grupo de edad.

Diversos estudios han informado una alta frecuencia (67\%) de co-infección entre $C$. trachomatis y $N$. gonorrhoea $e^{1,18}$. A pesar de lo anterior, otros estudios indican que ésta puede variar drásticamente, dependiendo del contexto y la prevalencia de fondo de cada una de estas infecciones. Por ejemplo, Creighton y cols. ${ }^{18}$, informaron que $38 \%$ de las mujeres con gonorrea también tenían clamidia. Sin

\begin{tabular}{|c|c|c|c|c|c|}
\hline \multirow[b]{2}{*}{$\begin{array}{l}\text { Edad } \\
\text { (años) }\end{array}$} & \multicolumn{5}{|c|}{ Chlamydia trachomatis } \\
\hline & $\begin{array}{c}\text { Total } \\
\text { pacientes }\end{array}$ & Positivo & Negativo & $95 \% \mathrm{Cl}$ & $\mathrm{p}<$ \\
\hline$\leq 14$ & 33 & $2(6,1 \%)$ & 31 & $1,8 \quad(0,43-7,44)$ & $N S^{b}$ \\
\hline $15-19$ & 238 & $26(10,9 \%)$ & 212 & $3,23(1,79-5,84$ & 0,001 \\
\hline $20-24$ & 233 & $13(5,6 \%)$ & 220 & $1,65(0,82-3,34)$ & NS \\
\hline $25-29$ & 475 & $17(3,6 \%)$ & 458 & $1,06(0,55-2,05)$ & NS \\
\hline $30-34$ & 789 & $25 \quad(3,2 \%)$ & 764 & $0,94(0,51-1,72)$ & NS \\
\hline $35-39^{a}$ & 503 & $17 \quad(3,4 \%)$ & 486 & $0,94(0,55-1,59)$ & NS \\
\hline$\geq 40$ & 81 & $2(2,5 \%)$ & 79 & $0,73(0,17-3,1)$ & NS \\
\hline
\end{tabular}

embargo, este porcentaje es menos frecuente en aquellas mujeres que están infectadas con C. trachomatis; sólo $13 \%$ de estas mujeres mostraron gonorrea, fenómeno también observado en varones heterosexuales. Estos datos sugieren que la adquisición de una infección gonocóccica reactiva una infección persistente por Chlamydia o aumenta la susceptibilidad del hospedero a la infección por clamidia.

Una limitación de este estudio es que no se analizó la co-infección de C. trachomatis con otros patógenos. Lo anterior fue debido a que las mujeres con datos clínicos de infección vaginal o con aislados positivos a otros microrganismos habían sido tratadas con antimicrobianos previamente siendo un criterio de exclusión en este protocolo.

Cabe señalar que los hallazgos clínicos de infección por clamidia deben corroborarse con las investigaciones de laboratorio ya que los signos y síntomas de otros patógenos como N. gonorrhoeae son indistinguibles. En este estudio solo se identifican aquellas mujeres que tienen infección por $C$. trachomatis y no otro patógeno, lo que podría condicionar cierto sesgo al eliminar las pacientes con infección por gonorrea; sin embargo, en esta inves- 
tigación no hubo aislados positivos para $N$. gonorrhoeae (datos no mostrados). Este resultado se puede corroborar con el número de mujeres con gonorrea que han sido reportados por otros hospitales de México en el Boletín Epidemiológico del Sistema Nacional de Vigilancia. Para la semana 52 de 2015 la infección por gonorrea sólo se detectó en Ciudad de México en tres casos en mujeres y 15 casos en hombres ${ }^{19}$.

Las adolescentes han sido consideradas como el grupo de mayor riesgo para adquirir la infección por C. trachomatis $^{1}$. La OMS define adolescencia como el período de edad entre 10 y 19 años $^{20}$. En esta investigación las adolescentes entre 15 y 19 mostraron una prevalencia similar a la de otros países como Noruega ${ }^{21}$, Estados Unidos de América (E.U.A. $)^{22}$, Reino Unido ${ }^{23,24}$, Perú ${ }^{25} \mathrm{e}$ Italia $^{26}$. Sin embargo, cabe señalar que todas las adolescentes estudiadas estaban embarazadas y con presencia de secreciones vaginales.

En México, la prevalencia de infección genital por clamidia en mujeres embarazadas es completamente desconocida, pero en otros países se ha informado que es entre 3,2 y $12,3 \%$, mientras que en adolescentes embarazadas se registra $14 \%{ }^{27,28}$. Es importante enfatizar que la detección oportuna de la infección por este patógeno puede prevenir las complicaciones que podría tener el recién nacido como la conjuntivitis, la neumonía o la sepsis ${ }^{14}$.

En este estudio, la prevalencia de infección en mujeres embarazadas fue de $6,7 \%$. En el caso de adolescentes embarazadas fue de $4,7 \%$ mientras que en las mujeres adultas fue de $2,0 \%$, esta diferencia posiblemente radique en los cuidados que tienen las parejas infértiles para no adquirir una ITS al desear tener un hijo.

En cuanto a los estudios sero-epidemiológicos de anticuerpos IgG anti-C. trachomatis en las mujeres embarazadas mexicanas, se comunican prevalencias de 22,6 a $77 \%$. Cabe señalar, que puede estar presente anticuerpos IgG contra otras especies de clamidia tales como C. psittaci y C. pneumoniae (reactividad cruzada), por lo que puede haber un alto número de falsas positivas, tal y como lo demostraron Hernández-Trejo y cols., quienes informaron que únicamente $0,9 \%$ mostraron anticuerpos sólo contra $C$. trachomatis de $85 / 110$ pacientes embarazadas seropositivas al género Chlamydia ${ }^{29}$.

Para disminuir la prevalencia de infección por $C$. trachomatis, el Centro de Control y Prevención de Enfermedades (CDC) de E.U.A., recomienda que todas las mujeres bajo 25 años de edad, que son sexualmente activas, deben someterse a pruebas rutinarias para el diagnóstico de infección por este patógeno para reducir los casos de EIP $^{30}$

En este estudio, la prevalencia de $C$. trachomatis en mujeres bajo 25 años (entre 20 y 24 años) fue inferior a $6 \%$; sin embargo, estas mujeres ya mostraron secuelas de infección al tener incapacidad para embarazarse pues se encontraron dentro del grupo de infertilidad. En este estudio, la mayor población de mujeres fueron las infértiles con edades entre 30 y 39 años, con una prevalencia de infección por clamidia no mayor a 3,5\%. Un estudio publicado en el año 2001 por nuestro grupo de investigación informó una prevalencia de $2,0 \%$ en las mujeres infértiles cuyos compañeros también fueron positivos para la infección por C. trachomatis ( $\mathrm{RR}=10,57$, IC del 95\% $5,67-19,7)^{2}$. Esto sugiere que ha habido un aumento de la prevalencia de infección por clamidia en las mujeres infértiles que asisten al INPer. Sin embargo, es importante señalar que también la diferencia en estos porcentajes se puede deber al método utilizado para el diagnóstico de este microorganismo. El método utilizado en el estudio del 2001 fue la hibridación en fase líquida (Gen-Probe PACE-2TM) que muestra una sensibilidad (75-85\%) menor al método de RPC-TR ${ }^{15}$.

Se ha descrito que aproximadamente $60 \%$ de las mujeres infértiles tienen anticuerpos IgG anti-C. trachomatis $^{1,5,15}$. Sin embargo, la detección de este patógeno genital es mucho menor siendo en algunos casos cercano a $30 \%{ }^{1,5,15}$; lo anterior sugiere un alto número de falsos negativos cuando el diagnóstico se realiza sólo con muestra endocervical. Esto ha sido confirmado en un estudio reciente por Villagrana y cols., quienes informaron que $20 \%$ de las pacientes con muestras endocervicales negativas para $C$. trachomatis realmente eran positivas a este patógeno cuando el diagnóstico se realizó con muestras de biopsia de endometrio o líquido peritoneal ${ }^{31}$.

Varios estudios han reportado el alto riesgo de desarrollar infertilidad si no se efectúa un diagnóstico y/o tratamiento temprano ${ }^{1,2,15}$. Por ejemplo, aproximadamente $4 \%$ de las mujeres con infección por $C$. trachomatis desarrollarán dolor pélvico crónico y $20 \%$ EIP. El resultado final en estas pacientes será una oclusión de las trompas de Falopio que se traducirá en infertilidad ${ }^{1,2,15}$.

Concluimos que la infección por C. trachomatis en las adolescentes está presente de manera importante lo que significa que posiblemente las campañas de salud sexual que está llevando a cabo el Ministerio de Salud en México sobre el uso de preservativos para un sexo seguro (para evitar embarazos no deseados e ITS) no han sido muy eficaces, aunque para confirmarlo se requiere llevar a cabo otro tipo de estudios.

Debido a lo anterior, se hace imperioso realizar un rastreo sistemático de las ITS en mujeres bajo 24 años de edad con vida sexual activa para disminuir los casos de EIP y de infertilidad, así como en aquellas mujeres embarazadas para prevenir las complicaciones de infección perinatal.

\section{Resumen}

Introducción: La infección endocervical por Chla- 
mydia trachomatis es considerada una de las principales causas de infertilidad en todo el mundo. Durante el embarazo puede conducir a complicaciones graves como la ruptura prematura de membranas y los partos prematuros. Objetivo: Determinar la prevalencia de infección genital por $C$. trachomatis en mujeres embarazadas e infértiles de la Ciudad de México. Métodos: La detección de $C$. trachomatis fue mediante reacción de polimerasa en cadena tiempo real (RPC-TR) con el kit comercial COBAS $^{\circledR}$ TaqMan CT Test v2,0 (Roche Molecular System). Resultados: Se analizaron 2.352 muestras; 102 fueron positivas $(4,3 \%)$. La prevalencia por edad mostró que las adolescentes embarazadas (15 a 19 años) fueron las de mayor riesgo de infección ( $10,9 \%, \mathrm{RR}=3,23$ [IC 95\%: $1,79-5,84])$, seguido de mujeres jóvenes de 20 a 24 años, con prevalencia de 5,6\% $(\mathrm{RR}=1,65$ [IC 95\%: 0,82-3,34]). Discusión: Los resultados indican que la prevalencia está dentro del rango reportado en el concierto mundial. Sin embargo, las adolescentes embarazadas tuvieron mayor prevalencia que las mujeres infértiles. Conclusión: Es imperioso realizar un rastreo sistemático de infección por $C$. trachomatis en mujeres bajo 24 años de edad, y en mujeres embarazadas para disminuir los casos de infertilidad y las complicaciones perinatales.

\section{Referencias bibliográficas}

1.- Malhotra M, Sood S, Mukherjee A, Muralidhar S, Bala M. Genital Chlamydia trachomatis: an update. Indian J Med Res 2013; 138: 303-16.

2.- Guerra-Infante F, Flores-Medina S, ArteagaTroncoso G, Zamora-Ruiz A, López-Hurtado M, Ortiz-Ibarra F J. Factores de riesgo y secuelas reproductivas asociados a la infección por Chlamydia trachomatis en mujeres infértiles. Salud Publica Mex 2003;45 Supl 5: S672-80.

3.- Guerra-Infante F M, Tapia-Yáñez J R, LópezHurtado M, Flores-Medina S, Díaz-García F J. Infección por Chlamydia trachomatis en varones y su asociación con las alteraciones ginecológicas de su compañera sexual. Rev Invest Clin 2005; 5: 406-14.

4.- de Jesus de Haro-Cruz M, Deleón-Rodriguez I, Escobedo-Guerra M R, López-Hurtado M, Arteaga-Troncoso G, Ortiz-Ibarra F J, et al. Genotyping of Chlamydia trachomatis from endocervical specimens of infertile Mexican women. Enferm Infec Microbiol Clin 2011; 29: 102-8. doi:10.1016/j.eimc.2010.08.014.

5.- Guerra Infante F M, López Hurtado M. Seroprevalencia de Chlamydia trachomatis en mujeres mexicanas. Salud Publica Mex 2004; 46: 100-1.

6.- Casillas-Vega N, Morfín-Otero R, García $\mathrm{S}$, Llaca-Díaz J, Rodríguez-Noriega E, Camacho-Ortiz A, Frequency and genotypes of Chlamydia trachomatis in patients attending the obstetrics and gynecology clinics in Jalisco, Mexico and correlation with sociodemographic, behavioral, and biological factors. BMC Womens Health 2017; 17: 83-91. doi: 10.1186/ s12905-017-0428-5.

7.- Echániz-Avilés G, Calderón-Jaimes E, Carnalla-Barajas N, Soto-Noguerón A, CruzValdez A, Gatica-Marquina R. Prevalence de infección cervicovaginal causada por Chlamydia trachomatis en población femenina de la ciudad de Cuernavaca, Morelos. Salud Publica Mex 1992; 34: 301-7.
8.- Acosta-Cázares B, Ruiz-Maya L, Escobedo de la Peña J. Prevalence and risk factors for Chlamydia trachomatis infection in low-income rural and suburban populations of Mexico. Sex Transm Dis 1996; 23: 283-8.

9.- Baldwin S B, Djambazov B, Papenfuss M, Abrahamsen M, Denman C, Guernsey de Zapien J, et al. Chlamydial infection in women along the US-Mexico border. Int J STD AIDS. 2004; 15: 815-21. DOI: $10.1258 / 0956462042563792$.

10.- Magaña-Contreras M, Contreras-Paredes A, Chavez-Blanco A, Lizano M, De la CruzHernandez Y, De la Cruz-Hernandez E. Prevalence of sexually transmitted pathogens associated with HPV infection in cervical samples in a Mexican population. J Med Virol. 2015; 87: 2098-105. doi: 10.1002/jmv.24278.

11.- Canto-de Cetina T, Polanco-Reyes L, Fernández-González V, Ruiz-García S. Infección por Chlamydia trachomatis en usuarias de dos clínicas de planificación familiar. Salud Publica Mex. 2003;45 Supl 5: S657-61.

12.- Uribe-Salas F, Conde-Glez C J, Juárez-Figueroa L, Hernández-Castellanos A. Sociodemographic dynamics and sexually transmitted infections in female sex workers at the Mexican-Guatemalan border. Sex Transm Dis 2003; 30: 266-71.

13.- Alvarado-Esquivel C, García-Villanueva A, Castruita-Limones D E, Cardosa-Nevárez F J, Ruiz-Astorga R. Prevalencia de infección por Chlamydia trachomatis en prostitutas registradas en la ciudad de Durango, México. Salud Publica Mex 2000; 42: 43-7.

14.- López-Hurtado M Guerra-Infante F M. Early neonatal infection by Chlamydia trachomatis J Infect Dis Ther. 2014; 2: 158. DOI: 10.4172/2332-0877.1000158.

15.- Land J A, Van Bergen J E, Morre S A, Postma M J. Epidemiology of Chlamydia trachomatis infection in women and the cost-effectiveness of screening. Hum Reprod Update. 2010; 16: 189-204. doi: 10.1093/humupd/dmp035.

16.- World Health Organization. Global incidence and prevalence of selected sexually transmitted infections-2008. Available at: www.who. int/reproductivehealth/publications/rtis/ stisestimates/en/. 2012 (Consultado el 9 noviembre de 2016).

17.- Newman L, Rowley J, Vander Hoorn S Wijesooriya N S, Unemo M, Low N, et al. Global estimates of the prevalence and incidence of four curable sexually transmitted infections in 2012 based on systematic review and global reporting. PloS one. 2015; 10: e0143304. doi: 10.1371/journal.pone.0143304.

18.- Creighton S, Tenant-Flowers M, Taylor C B, Miller R, Low N. Co-infection with gonorrhoea and chlamydia: how much is there and what does it mean? Int J STD AIDS. 2003; 14: 10913. DOI: $10.1258 / 095646203321156872$.

19.- Boletín Epidemiológico del Sistema Nacional de Vigilancia www.gob.mx/cms/uploads/ attachment/file/50233/sem52.pdf (Consultado el 3 de octubre de 2017).

20.- WHO. Adolescent development. www.who.int/maternal_child_adolescent/ topics/adolescence/dev/en/ (Consultado el 9 noviembre de 2016).

21.- Klovstad H, Aavitsland P. Chlamydia trachomatis infections in Norway, 1986 to 2006, surveillance data. Sex Transm Dis 2009; 36: 17-21. doi: 10.1097/ OLQ.0b013e31818653b0.

22.- Morbidity and Mortality Weekly Report. Chlamydia screening among sexually active young female enrollees of health plans-United States, 1999-2001. Morbid Mortal Wkly Rep MMWR. 2004; 53: 983-5. https://www.cdc. gov/mmwr/preview/mmwrhtml/mm5814a2. htm.

23.- Adams E J, Charlett A, Edmunds W J, Hughes G. Chlamydia trachomatis in the United Kingdom: a systematic review and analysis of prevalence studies. Sex Transm Infect. 2004; 80: 354-62. DOI: 10.1136/ sti.2003.005454

24.- Scott L D, Baster K, Emmett L, Nichols $\mathrm{T}$, Randall S, McLean L, et al. Incidence 
and reinfection rates of genital chlamydial infection among women aged 16-24 years attending general practice, family planning and genitourinary medicine clinics in England: a prospective cohort study by the Chlamydia Recall Study Advisory Group. Sex Transm Infect 2007; 83: 292-303. doi: 10.1136/ sti.2006.022053.

25.- Paul K J, García P J, Giesel A E, Holmes K K, Hitti JE. Generation C: prevalence of and risk factors for Chlamydia trachomatis among adolescents and young women in Lima, Peru. J Womens Health (Larchmt) 2009; 18: 1419-24. doi: 10.1089/jwh.2008.1069.

26.- Marcone V, Recine N, Gallinelli C, Nicosia R, Lichtner M, Degener A M, et al. Epidemiology of Chlamydia trachomatis endocervical infection in a previously unscreened population in Rome, Italy, 2000 to 2009. Euro Surveill. 2012;17: pii 20203. https://doi.org/10.2807/ ese.17.25.20203-en.

27.- Silveira M F, Ghanem K G, Erbelding E J, Burke A E, Johnson H L, Singh R H, et al. Chlamydia trachomatis infection during pregnancy and the risk of preterm birth: a casecontrol study. Int J STD AIDS 2009, 20 (7): 465-9. doi: 10.1258/ijsa.2008.008388.

28.- Silveira M F, Sclowitz I K, Entiauspe L G, Mesenburg M A, Stauffert D, Bicca G L, Pet al. Chlamydia trachomatis infection in young pregnant women in Southern Brazil: a crosssectional study. Cad Saude Publica 2017; 33 : e00067415. doi: 10.1590/0102-311X00067415. 29.- Hernández-Trejo M, Herrera-González N,
Guerra-Infante F M. Evidencia serológica de infección por tres especies de Chlamydia en mujeres embarazadas. Ginecol Obstet Mex 2014; 82: 585-90.

30.- Centers for Disease Control and Prevention. Sexually Transmitted Diseases Treatment Guidelines, 2015. Morb Mortal Wkly Rep (MMWR). http://www.cdc.gov/mmwr/preview/ mmwrhtml/rr6403a1.htm. (Consultado el $9 \mathrm{de}$ noviembre de 2016).

31.- Villagrana-Zesati J R, López-Hurtado M, Flores-Salazar V R, de Haro-Cruz M J, Escobedo-Guerra M R, Guerra-Infante F M. Persistence of Chlamydia trachomatis in endometrium, and peritoneal fluid of infertile patients with negative cervical culture. Ginecol Obstet Mex 2013; 81: 23-8. 